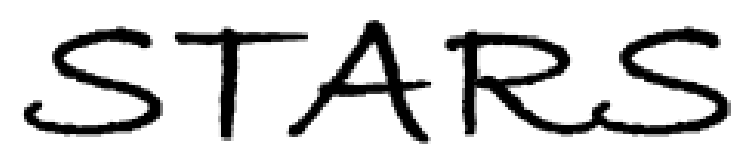

University of Central Florida

STARS

Faculty Bibliography 2000s

Faculty Bibliography

$1-1-2007$

\title{
Two-dimensional discrete Ginzburg-Landau solitons
}

Nikolaos K. Efremidis

Demetrios N. Christodoulides

University of Central Florida

Kyriakos Hizanidis

Find similar works at: https://stars.library.ucf.edu/facultybib2000

University of Central Florida Libraries http://library.ucf.edu

This Article is brought to you for free and open access by the Faculty Bibliography at STARS. It has been accepted for inclusion in Faculty Bibliography 2000s by an authorized administrator of STARS. For more information, please contactSTARS@ucf.edu.

\section{Recommended Citation}

Efremidis, Nikolaos K.; Christodoulides, Demetrios N.; and Hizanidis, Kyriakos, "Two-dimensional discrete Ginzburg-Landau solitons" (2007). Faculty Bibliography 2000 s. 7094.

https://stars.library.ucf.edu/facultybib2000/7094

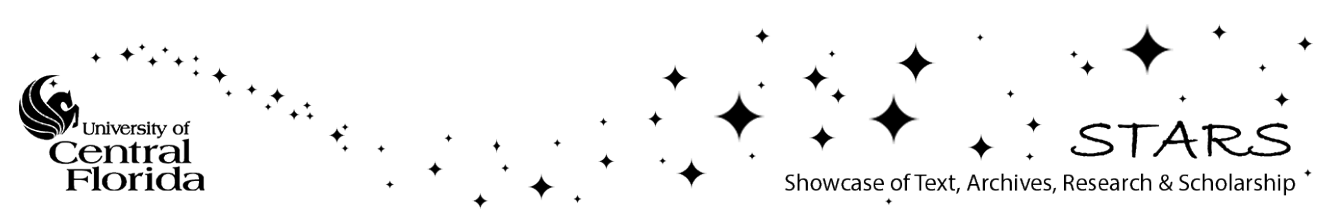




\title{
Two-dimensional discrete Ginzburg-Landau solitons
}

\author{
Nikolaos K. Efremidis, ${ }^{1}$ Demetrios N. Christodoulides, ${ }^{2}$ and Kyriakos Hizanidis ${ }^{3}$ \\ ${ }^{1}$ Department of Applied Mathematics, University of Crete, 71409 Heraklion, Crete, Greece \\ ${ }^{2}$ College of Optics and Photonics, University of Central Florida, Orlando, Florida 32813, USA \\ ${ }^{3}$ School of Electrical and Computer Engineering, National Technical University of Athens, Athens 15773, Greece
}

(Received 31 August 2007; published 25 October 2007)

\begin{abstract}
We study the two-dimensional discrete Ginzburg-Landau equation. In the linear limit, the dispersion and gain curves as well as the diffraction pattern are determined analytically. In the nonlinear case, families of two-dimensional discrete solitons are found numerically as well as approximately in the high-confinement limit. The instability dynamics are analyzed by direct simulations.
\end{abstract}

DOI: 10.1103/PhysRevA.76.043839

PACS number(s): 42.65.Tg, 42.65.Jx

\section{INTRODUCTION}

The complex Ginzburg-Landau (GL) equation is a ubiquitous model in nature. It appears in diverse branches of science such as, for example, superconductivity and superfluidity, nonequilibrium fluid dynamics and chemical systems, nonlinear optics, Bose-Einstein condensates, and quantum field theories [1-3]. Localized solutions or dissipative solitons of the GL equation are possible as a result of the interplay between gain and loss, complex diffraction (or dispersion), and nonlinearity [3-6].

On the other hand, the study of discrete or periodic optical systems has attracted a lot of attention during recent years. There are two main reasons for this growth: the development of experimental techniques making it possible to realize experiments in complicated periodic nonlinear structures and the potential for all-optical switching applications [7]. Optical discrete solitons were predicted in [8] and experimentally observed a decade later [9]. The transition from onedimensional to two-dimensional periodic systems came a few years later, and two-dimensional lattice solitons were observed in Ref. [10] using an optical induction technique $[11,12]$. In the coupled mode theory limit, optical periodic systems can be studied using the discrete nonlinear Schrödinger equation (DNLS).

The presence of gain or loss, due to optical amplifiers and/or saturable absorbers, in waveguide arrays leads to more complicated dynamics as compared to the conservative limit. Such nonconservative systems with a periodic index modulation can be modeled by a discrete Ginzburg-Landau (DGL) equation. DGL-like systems have been considered in semiconductor laser arrays [13-15], and in connection with spatiotemporal chaos, instabilities, and turbulence [16,17]. In addition, DGL lattices can also model Taylor and frustrated vortices in hydrodynamics [18].

Spatially localized dissipative discrete solitons of the onedimensional DGL lattices have been studied in Ref. [19] (see also [20] for a review). In that work, the linear properties as well as the families of dissipative soliton solutions were analyzed numerically and asymptotically. In the linear limit, Bloch oscillations are also possible in lattices that have a linearly varying propagation constant [21]. These oscillations occur in spite of the fact that the Bloch wave packet experiences coupling gain and (or) loss.
Experimental results have shown that, in multicore fiber laser arrays, the in-phase or out-of-phase mode is selectively amplified according to the self-phase-modulation coefficient [22]. The existence and stability of nonlinear modes in centrally coupled circular arrays of waveguides was considered in Ref. [23].

In Ref. [24] it is shown that dissipative optical lattice solitons are possible in waveguide configurations that involve periodically patterned semiconductor optical amplifiers and saturable absorbers.

Exact bright soliton solutions are not known to exist for the DGL equation. However, if one considers an AblowitzLadik [25] type of discretization of the GL equation, exact solutions can be found in both nonlocal cubic [26] and nonlocal quintic nonlinearities [27]. In Ref. [28] solitons in a perturbed Ablowitz-Ladik discrete NLS equation are considered. The perturbations taken into account include linear dumping, nonlinear cubic amplification and quintic dumping, and the complex second difference.

Dissipative discrete solitons have also been studied in arrays of coupled waveguide resonators [29]. Modulational instability and generation of nonlinear modulated waves was also investigated for the DGL equation [30,31].

Up to now, in the area of dissipative optical lattices, only one dimensional topologies have been investigated. Here, we consider a two-dimensional dissipative waveguide lattice which is modeled by a two-dimensional DGL equation. We study the linear discrete diffraction and gain properties of the system. In the nonlinear case, families of dissipative discrete soliton solutions are identified, both numerically and asymptotically in the case of high confinement. The stability of the solutions is tested numerically. In the long-wavelength limit we also demonstrate that the two-dimensional DGL equation can be approximated by a two-dimensional GL equation.

\section{MODEL}

Let us start by writing the two-dimensional discrete Ginzburg-Landau equation:

$$
i \dot{u}_{j, k}-i \epsilon u_{j, k}+\alpha \Delta u_{j, k}+p\left|u_{j, k}\right|^{2} u_{j, k}+q\left|u_{j, k}\right|^{4} u_{j, k}=0,
$$

where $\Delta u_{j, k}=u_{j+1, k}+u_{j-1, k}+u_{j, k+1}+u_{j, k-1}$ is the discrete diffraction operator that accounts for nearest-neighbor couplings in a two-dimensional square lattice. The parameters $\alpha$, 
$p$, and $q$ are in general complex and can be expressed in terms of real variables as $\alpha=\alpha_{r}+i \alpha_{i}, p=p_{r}+i p_{i}$, and $q=q_{r}$ $+i q_{i}$, whereas $\epsilon$ is real. In Eq. (1) $\epsilon$ accounts for linear gain or loss and $p_{i}, q_{i}$ represent the nonlinear gains and/or losses of the system. $p_{r}$ and $q_{r}$ are the coefficients of the cubic and quintic nonlinearities. The coefficient $\alpha_{r}$ stands for the linear coupling among nearest neighbors, and $\alpha_{i}$ corresponds to gain or loss due to coupling.

\section{LINEAR PROPERTIES}

The diffraction and gain curves of the system can be obtained by assuming low-amplitude plane wave solutions having the form

$$
u_{j, k}=\exp \left[i q z-i\left(\theta_{x} j+\theta_{y} k\right)\right]
$$

where $q=q_{r}+i q_{i}$. In Eq. (2) $\theta_{x}$ and $\theta_{y}$ are real, and represent the Bloch momentum along the $x$ and $y$ directions. Experimentally these parameters can be adjusted by changing the input tilt of the laser beam inside the array. Substituting Eq. (2) into the linear part of the DGL equation (1) and separating the real and imaginary parts, the diffraction

$$
q_{r}=2 \alpha_{r}\left(\cos \theta_{x}+\cos \theta_{y}\right)
$$

and gain (or diffusion)

$$
q_{i}=2 \alpha_{i}\left(\cos \theta_{x}+\cos \theta_{y}\right)-\epsilon
$$

relations are obtained.

From Eq. (3) and assuming a positive coupling coefficient $\alpha_{r}>0$, the second order diffraction along the $j=\{x, y\}$ direction (given by $2 \alpha_{r} \cos \theta_{j}$ ) is normal when $-\pi / 2<\theta_{j}<\pi / 2$ and anomalous for $\pi / 2<\left|\theta_{j}\right|<\pi$. Thus, the diffraction relation is of the elliptic type when $\cos \theta_{x} \cot \theta_{y}>0$ and hyperbolic when $\cos \theta_{x} \cos \theta_{y}<0$. Utilizing the dispersion relation, the group velocity of a linear input beam is derived:

$$
\mathbf{v}_{g}=-\nabla_{\theta} q_{r}=2 \alpha_{r}\left(\sin \theta_{x} \hat{\mathbf{x}}+\sin \theta_{y} \hat{\mathbf{y}}\right) .
$$

On the other hand, the growth rate of a low-intensity (linear) signal is given by $g=-q_{i}$. Thus, the discrete Fourier components of an input beam for which $g\left(\theta_{x}, \theta_{y}\right)>0(<0)$ are going to experience growth (decay) along $z$. A necessary condition for the stability of a nonlinear spatially localized beam is the stability of the supporting zero background. The zero solution is stable when $g\left(\theta_{x}, \theta_{y}\right)<0$ for all possible values of $\theta_{x}$ and $\theta_{y}$ inside the first Brillouin zone. This latter condition results in

$$
\epsilon<-4\left|\alpha_{i}\right|
$$

The linear diffraction or diffusion pattern, when a single lattice site is excited at the input $\left[u_{j, k}(0)=\delta_{j, 0} \delta_{k, 0} u_{0}\right]$ is derived from the following formula:

$$
u_{j, k}(z)=u_{0} J_{j}(2 \alpha z) J_{k}(2 \alpha z) e^{i \pi(j+k) / 2} e^{i \epsilon z} .
$$

Furthermore, the equation for diffraction or diffusion of an arbitrary initial condition can be solved exactly by appropriately superimposing Eq. (7). In Fig. 1 the linear diffraction pattern resulting from a single waveguide excitation is shown for different values of the parameters at $z=1.5$. In Fig.

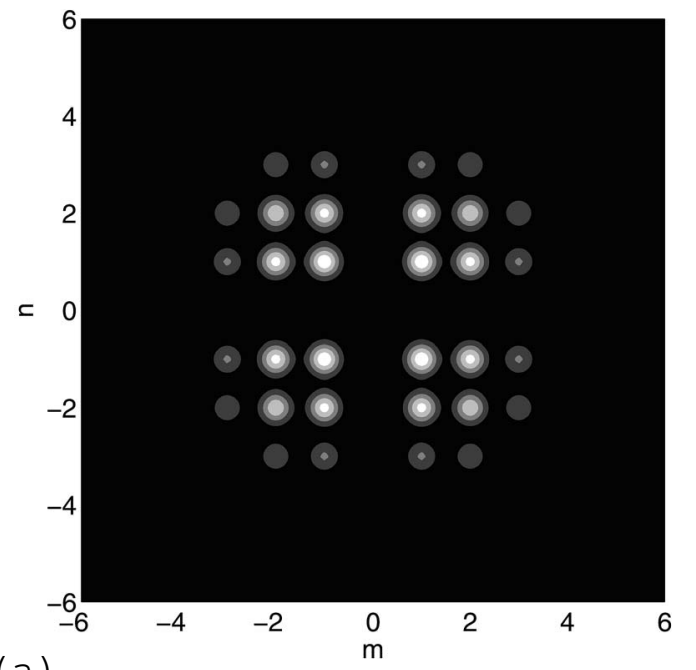

(a)

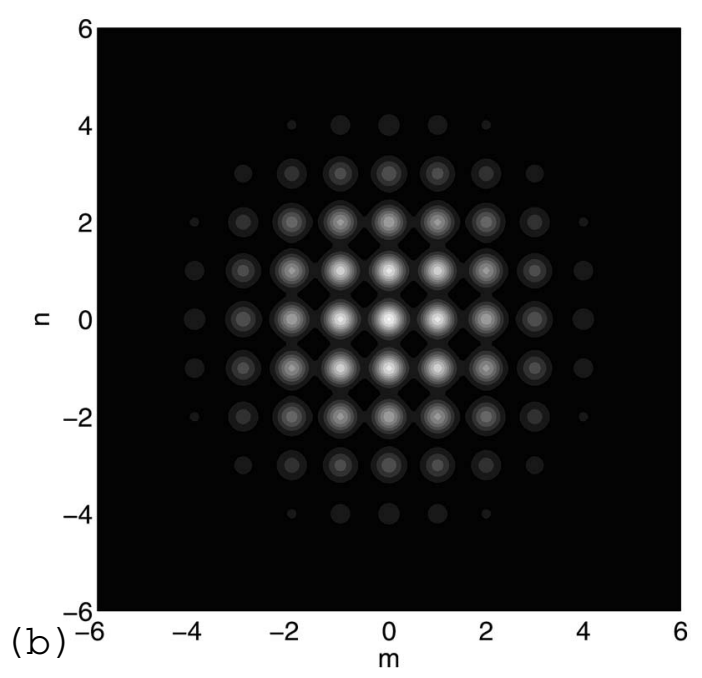

FIG. 1. Linear diffraction field amplitude pattern [given by Eq. (7)] as obtained for $\epsilon=0$ at $z=1.5$ for $\alpha=1$ (a) and (b) $1+i$. The initial condition corresponds to single (central) waveguide excitation.

1(a) the conservative limit is taken $(\alpha=1)$. As is graphically depicted, the diffraction concentrates most of the intensity at the perimeter of a square. On the other hand, in the nonconservative limit $\alpha=i \alpha_{i}$, the beam intensity maintains a Gaussian-like profile along $z$. When $\alpha_{i}>0$ in-phase modes are preferably amplified, whereas for $\alpha_{i}<0$ the field pattern becomes $\pi$ out of phase as it evolves. In Fig. 1(b), $\alpha=1+i$, gain dominates, and at the output the field amplitude has a bell shape.

An exact formula for the field evolution can also be derived when the propagation constant varies linearly across the array. In that case, the input beam experiences Bloch oscillations in spite of the fact that the Bloch wavepacket exhibits gain or loss [21].

The parameter space for which standing DGL solitons bifurcate (via a Hopf bifurcation) from the zero solution can be found by isolating the points for which the growth rate becomes zero, $g\left(\theta_{x}, \theta_{y}\right)=0$, and in addition $\boldsymbol{\nabla}_{\theta} g=\mathbf{0}$. Three different such points exist, in particular, the $\Gamma\left(\theta_{x}=\theta_{y}=0\right)$, the $X$ $\left(\theta_{x}=0, \theta_{y}= \pm \pi\right.$ or $\left.\theta_{x}= \pm \pi, \theta_{y}=0\right)$, and the $M\left(\theta_{x}= \pm \pi, \theta_{y}\right.$ 
$= \pm \pi)$ points of the Brillouin zone. Notice that in the onedimensional case, two bifurcation points, located at the base and the edge of the Brillouin zone, exist [19].

\section{LONG-WAVELENGTH APPROXIMATION}

Let us express $u_{j, k}$ in terms of the amplitude and phase, $u_{j, k}=\rho_{j, k} \exp \left(i \phi_{j, k}\right)$. In the long-wavelength limit, it is assumed that the optical wave is broad enough that the amplitude difference between adjacent lattice sites is very small $\left(\left|\rho_{j \pm 1, k}-\rho_{j, k}\right| \ll 1\right.$ and $\left.\left|\rho_{j, k \pm 1}-\rho_{j, k}\right| \ll 1\right)$, and the corresponding phase differences in the $x$ and $y$ directions are almost constant, say $\theta_{x}$ and $\theta_{y}$, respectively $\left(\left|\phi_{j \pm 1, k}-\phi_{j, k}\right| \simeq \theta_{x}\right.$ and $\left.\left|\phi_{j, k \pm 1}-\phi_{j, k}\right| \simeq \theta_{y}\right)$. Then the phase transformation

$$
u_{j, k}=v_{j, k} \exp \left[i\left(\theta_{x} j+\theta_{y} k\right)\right]
$$

is applied to Eq. (1). We can consider $v_{j, k}$ as a discretized sample of the continuous function $v(x, y)$ with steps $\Delta x$ and $\Delta y$ in the $x$ and $y$ directions, i.e.,

$$
v_{j, k}=v\left(x_{j}, y_{k}\right),
$$

where

$$
x_{j}=j \Delta x, \quad x_{k}=k \Delta y .
$$

Without loss of generality, we can assume these steps to be unity, $\Delta x=\Delta y=1$. The linear coupling terms among nearest neighbors of the DGL equation can now be expressed by the following Taylor series around $v_{j, k}$ :

$$
\begin{aligned}
& v_{j \pm 1, k}=\sum_{l=0}^{\infty} \frac{( \pm 1)^{l}}{l !} \frac{\partial^{l} v\left(x_{j}, y_{k}\right)}{\partial x^{l}}, \\
& v_{j, k \pm 1}=\sum_{l=0}^{\infty} \frac{( \pm 1)^{l}}{l !} \frac{\partial^{l} v\left(x_{j}, y_{k}\right)}{\partial y^{l}} .
\end{aligned}
$$

Substituting the expansions (11) and (12) into Eq. (1), the following nonlinear complex Ginzburg-Landau equation with nonlocal complex diffraction term is obtained:

$$
\begin{aligned}
i \frac{\partial v}{\partial z}- & i \epsilon v+p|v|^{2} v+q|v|^{4} v+\frac{\alpha}{\pi} \iiint \int_{-\infty}^{\infty} d x^{\prime} d y^{\prime} d q_{x} d q_{y} \\
& \times \cos \left(\theta_{x}-q_{x}\right) \cos \left(\theta_{y}-q_{y}\right) e^{i\left[q_{x}(x \prime-x)+q_{y}(y \prime-y)\right]} v\left(x^{\prime}, y^{\prime}\right)=0 .
\end{aligned}
$$

Up to this point, no assumptions have been made about the form of the solution and thus Eq. (13) is equivalent to the DGL equation (1). From the phase transformation (8) in the long-wavelength limit of $v_{j, k}$, both the amplitude and the phase vary slowly with the lattice index and thus

$$
\left|v_{j \pm 1, k}-v_{j, k}\right| \ll 1, \quad\left|v_{j, k \pm 1}-v_{j, k}\right| \ll 1 .
$$

From Eq. (14) it is evident that one can obtain an accurate approximation of Eq. (13) by keeping up to second-order derivatives in Eqs. (11) and (12). Subsequently, the transformation $v=U \exp \left(i \alpha_{r} x\right)$ and the coordinate system change $X$ $=x-c_{x} z, Y=y-c_{y} z, Z=z$ are applied, resulting in the following cubic-quintic complex Ginzburg-Landau equation:

$$
i \frac{\partial U}{\partial Z}-i \epsilon^{\prime} U+\alpha_{x} \frac{\partial^{2} U}{\partial X^{2}}+\alpha_{y} \frac{\partial^{2} U}{\partial Y^{2}}+p|U|^{2} U+q|U|^{4} U=0,
$$

where

$$
\begin{gathered}
\epsilon^{\prime}=\epsilon-2 \alpha_{i}\left(\cos \theta_{x}+\cos \theta_{y}\right), \\
c_{x}=2 \alpha \sin \theta_{x}, \quad c_{y}=2 \alpha \sin \theta_{y} .
\end{gathered}
$$

and

$$
\alpha_{x}=\alpha \cos \theta_{x}, \quad \alpha_{y}=\alpha \cos \theta_{y} .
$$

\section{FAMILIES OF HIGHLY CONFINED DISSIPATIVE DISCRETE SOLITONS}

Families of highly confined DGL soliton solutions can be identified by utilizing the symmetries of the problem. Let us first assume that Eq. (1) admits solutions of the form $u_{j, k}$ $=v_{j, k} \exp (i q z)$ and thus

$$
-(q+i \epsilon) v_{j, k}+\alpha \Delta v_{j, k}+p\left|v_{j, k}\right|^{2} v_{j, k}+q\left|v_{j, k}\right|^{4} v_{j, k}=0 .
$$

Equation (19) follows a simple phase invariance. More specifically, if $v_{j, k}$ is a solution of Eq. (19), $v_{j, k} \exp (i \phi)$ is also a solution. This invariance can be used to consider solutions such that $v_{0,0}=A$ where $A$ is a positive number.

Let us focus on solutions that are highly confined in the lattice. We can assume that the amplitude of $v_{j, k}$ is $A$ on the maximum-intensity lattice sites, and $|\delta|$ ( $\delta$ being a complex number) on the lattice sites that are adjacent to the highintensity sites. Thus, the condition for high confinement results as $|\delta| \ll A$.

Notice that the solutions have specific symmetries according to their quadrant. For the sake of simplicity, let us analyze the symmetries in the one-dimensional case and then generalize these results for two-dimensional lattices. Such a symmetry analysis is adopted from linear discrete systems [32]. In one dimension the $z$-independent form of the DGL equation is

$$
L_{j} v_{j}=-(q+i \epsilon) v_{j}+\alpha \Delta v_{j}+F_{j} v_{j}=0,
$$

where $F_{j}=p\left|v_{j}\right|^{2}+q\left|v_{j}\right|^{4}$. First, we consider "on-site" soliton solutions such that (i) the field amplitude is maximum for lattice element 0 and decreases for increasing $|j|$ and (ii) the amplitude is an even function of $j$ with symmetry $\left|v_{j}\right|=\left|v_{-j}\right|$. From the second condition, $F_{j}=F_{-j}$, and thus $L_{-j} v_{j}=0$. Applying the transformation $j \rightarrow-j$ we find that

$$
L_{j} v_{-j}=0 .
$$

From Eqs. (20) and (21) the phase condition $v_{j}=v_{-j} \exp (i \theta)$ between positive and negative waveguides is obtained. However, since $u_{j}$ has a unique value for $j=0$, we find that $\theta=0$, i.e., $v_{-j}=v_{j}$ for soliton solutions with a single peak.

On the other hand, off-site discrete solitons have their maxima at two adjacent lattice sites, i.e., $\left|v_{0}\right|=\left|v_{1}\right|=A$, and, in addition, satisfy the even parity condition $\left|v_{j}\right|=\left|v_{1-j}\right|$ which 
TABLE I. Different families of two-dimensional dissipative solitons. Parameter $A$ is positive, whereas $\delta$ is in general complex and, in addition, $|\delta| \ll A$.

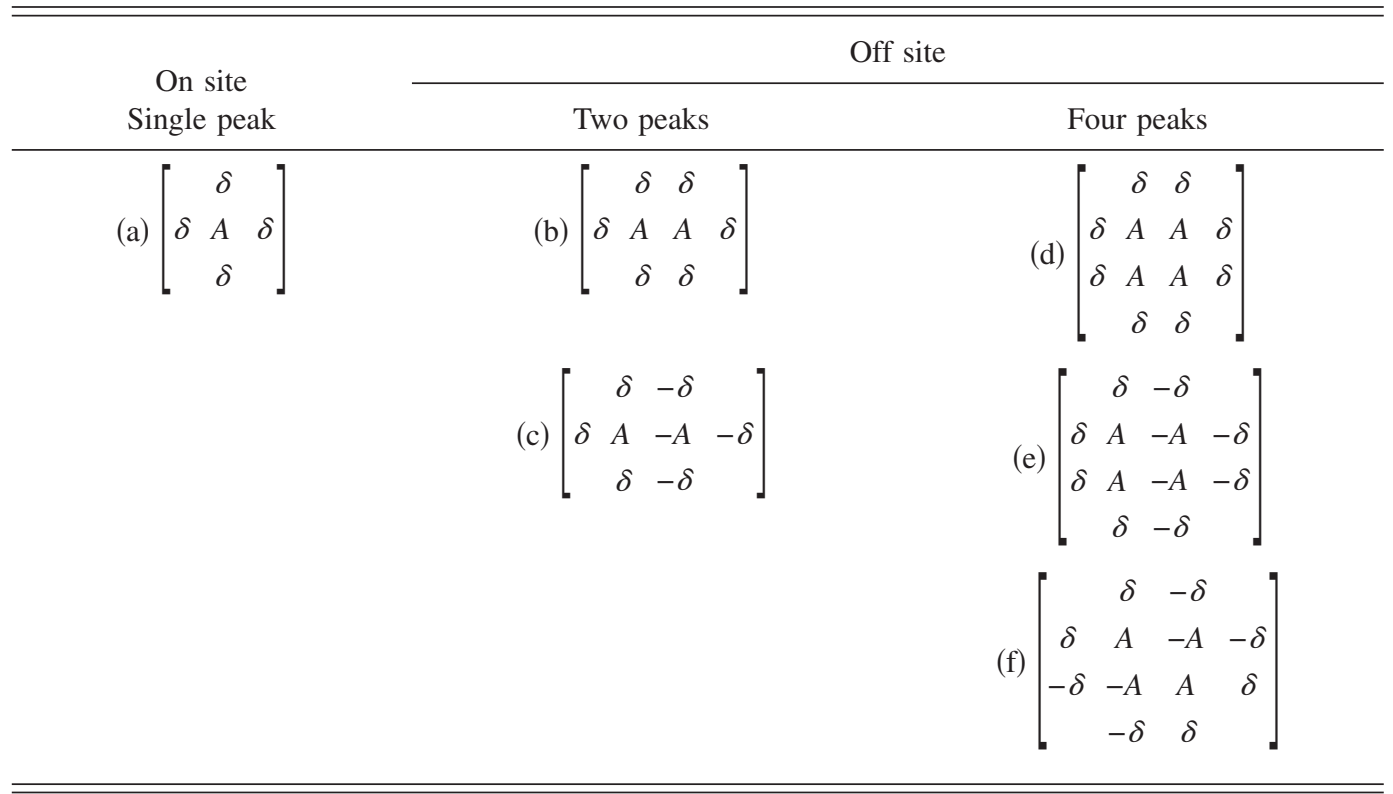

results in $L_{1-j} v_{j}=0$. Applying the transformation $j \rightarrow 1-j$, we obtain

$$
L_{j} v_{1-j}=0 .
$$

From Eqs. (20) and (22) it turns out that $v_{j}=v_{1-j} \exp (i \theta)$. Subtracting the equation $L_{1} v_{1}=0$ from $L_{0} v_{0}=0$ results in the phase condition $\exp (2 i \theta)=1$, or $\theta=0, \pi$. Thus, there is an exact 0 or $\pi$ phase difference between the high-amplitude lattice sites although the lattice parameters are complex.

Notice that, in the DNLS equation case, the phase difference between first neighbors $\left[\Delta \phi_{j}=\phi_{j+1}-\phi_{j}\right.$ where $v_{j}$ $\left.=\rho_{j} \exp \left(i \phi_{j}\right)\right]$ is the same for all values of $j$ and is equal to 0 or $\pi$. On the other hand, in dissipative DGL lattices the phase difference $\Delta \phi_{j}$ in general depends on $j$.

Similar symmetry arguments can be applied for twodimensional lattices. All the families of dissipative soliton solutions discussed here are shown in Table I. We are considering three different cases shown in the three columns of Table I. These soliton families are on-site which has a single intensity peak, the off-site with two peaks, and off-site with four high-intensity peaks.

Let us first consider the on-site solution whose amplitude has the symmetries $F_{j, k}=F_{-j, k}=F_{j,-k}=F_{-j,-k}$. Following a similar analysis as for the one-dimensional case, it can be shown that $u_{j, k}=u_{-j, k}=u_{j,-k}=u_{-j,-k}$. For off-site solutions with two peaks, we consider the intensity pattern with the parity $F_{i, j}=F_{1-i, j}=F_{i,-j}=F_{1-i,-j}$. In this case, two different possibilities for the field exist, which are shown in the second column of Table I. The quadrants of the first family of such solutions [Table I(b)] satisfy $u_{i, j}=u_{1-i, j}=u_{i,-j}=u_{1-i,-j}$ and thus the phase difference among the high-intensity elements is zero. The quadrants of the second family [Table $\mathrm{I}(\mathrm{c})]$ have the symmetry $u_{i, j}=u_{i,-j}=-u_{1-i, j}=-u_{1-i,-j}$ and, thus the phase difference between the high intensity sites is $\pi$. Finally, in the right column of Table I off-site solitons having four high- intensity peaks and even intensity symmetry $F_{i, j}=F_{1-i, j}$ $=F_{i, 1-j}=F_{1-i, 1-j}$ are shown. Following the algebra one can find that there are three nondegenerate cases for the field. In case (d), $u_{i, j}=u_{1-i, j} u_{i, 1-j}=u_{1-i, 1-j}$, for (e), $u_{i, j}=-u_{1-i, j}=u_{i, 1-j}$ $=-u_{1-i, 1-j}$, whereas for (f), $u_{i, j}=-u_{1-i, j}=-u_{i, 1-j}=u_{1-i, 1-j}$.

\section{APPROXIMATE DGL HIGHLY CONFINED SOLITONS}

Approximate analytic expressions for the dissipative DGL solitons shown in Table I can be found in the case of discrete modes that are highly localized inside the lattice. The highconfinement limit is widely used for finding approximate accurate solutions in conservative discrete lattices [33,34].

Let us start by considering the first family of solutions [Table I(a)] which has a maximum on a single lattice site. This family of solution is called on site because the maximum of an imaginary envelope is located exactly at the lattice site $(0,0)$. Following the algebra and assuming that $v_{0,0}=A \gg\left|v_{i, j}\right|$ where $(i, j) \neq(0,0)$, we get

$$
A^{2}=\frac{1}{2 q_{i}}\left(-p_{i} \pm \sqrt{p_{i}^{2}+4 q_{i} \epsilon}\right) .
$$

In Eq. (23) the amplitude as a function of the bifurcation parameter $\epsilon$ is obtained. Notice that the solutions of the DGL equation have codimension 1. Thus, for fixed $\epsilon$ only a finite discrete set (and not a family) of on-site solutions exists. More specifically, according to the values of the parameters, Eq. (23) supports zero, one, or two different soliton solutions, each one of them having a different amplitude. In addition, the soliton propagation constant is obtained from

$$
q=p_{r} A^{2}+q_{r} A^{4}
$$

Finally, the amplitude of the four lattice sites adjacent to the high-intensity central one is well approximated by 


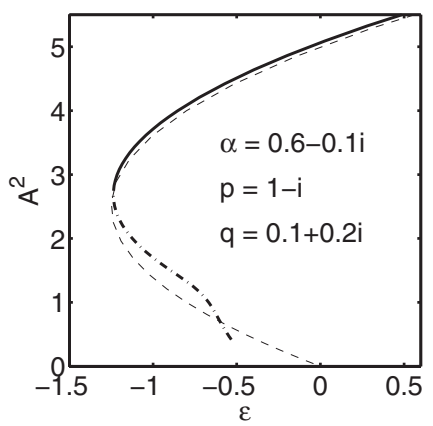

FIG. 2. Numerically obtained bifurcation curve of the on-site mode schematically shown in Table I(a) (thick solid and dashdotted curves). The solid and dash-dotted curves correspond to stable and unstable solutions, respectively. The approximate analytical expression for the curve in the case of high confinement [Eq. (23)] is depicted with a thin dashed curve. The parameters of the DGL equation can be found inside the figure.

$$
u_{i, j}=\delta=\frac{A \alpha}{q+i \epsilon}=\frac{A \alpha}{p_{r} A^{2}+q_{r} A^{4}+i \epsilon},
$$

where $\{i, j\}= \pm 1$. The necessary condition for high lattice confinement $|\delta / A| \ll 1$ using Eqs. (23) and (25) becomes

$$
\left|\frac{A \alpha}{p_{r} A^{2}+q_{r} A^{4}+i \epsilon}\right|^{2} \ll 1 .
$$

The same analysis can be repeated for all the families of solutions shown in Table I. For each of these families, there is a simple transformation that can be applied to Eqs. (23) and (24) to determine $A^{2}$ and $q$. Notice that parameter $\delta$ is always given by Eq. (25). Specifically, for the solutions shown in Table $\mathrm{I}(\mathrm{b})$ this change of variables is $\epsilon \rightarrow \epsilon-\alpha_{i}$ along with $q \rightarrow q-\alpha_{r}$. For the family (c) $A^{2}$ and $q$ are obtained through $\epsilon \rightarrow \epsilon+\alpha_{i}$ and $q \rightarrow q+2 \alpha_{r}$, For the solutions shown in Table I(d) the transformation is $\epsilon \rightarrow \epsilon-2 \alpha_{i}$ and $q$ $\rightarrow q-2 \alpha_{r}$, whereas for the families (e) and (f) Eqs. (23) and (24) remain unchanged.

The decay of soliton solutions for large values of $\sqrt{m^{2}+n^{2}}$ can be obtained asymptotically in the long-wavelength limit. In this case, the form of the solution at infinity becomes

$$
K_{0}\left[\left(\frac{q+i \epsilon}{\alpha}-4\right) \sqrt{m^{2}+n^{2}}\right],
$$

where $K_{0}$ is a modified Bessel function.

It is perhaps interesting to point out that, from the above analysis for highly confined solutions, no distinction is made between solutions residing at different points of the Brillouin zone. This happens because, up to the first-order approximation considered here, all three families of solutions become essentially identical in the strong-localization limit.

\section{NUMERICAL RESULTS}

Exact numerical solutions of the DGL equation were obtained using Newton's iteration scheme. Initial conditions for the numerics were provided by the asymptotic analysis of the (a)
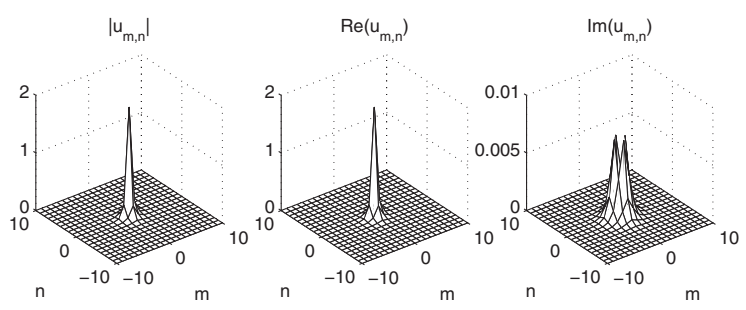

(b)
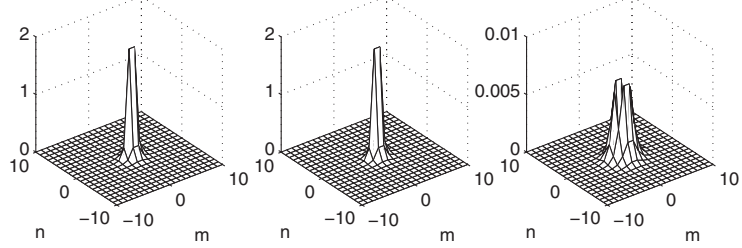

(c)
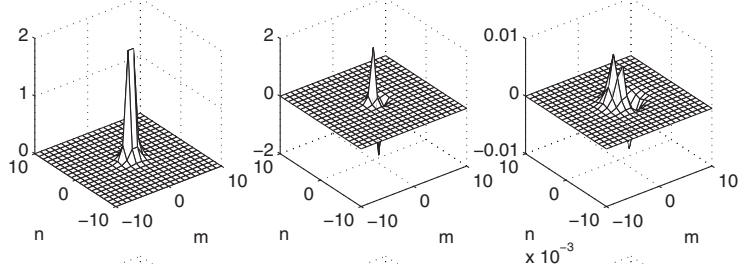

(d)
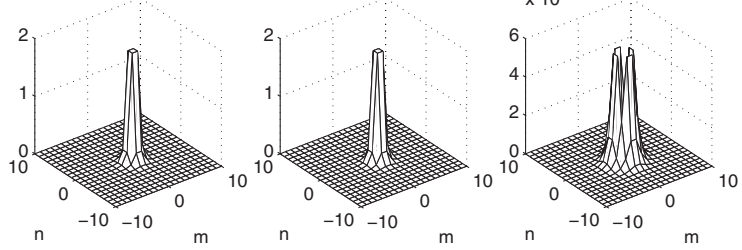

(e)
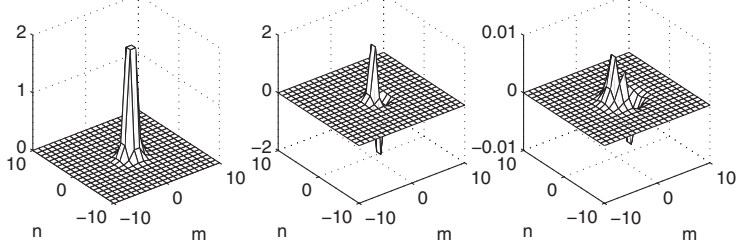

(f)
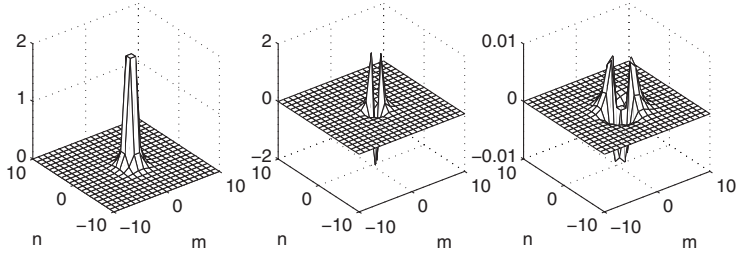

FIG. 3. Numerically obtained dissipative soliton solutions of the DGL equation. In the left, center, and right columns the field amplitude and the real and imaginary parts of the solution are depicted, respectively. These solutions have been chosen to have maximum amplitude $A=1.9$. Rows (a)-(f) correspond to solutions with the same index in Table I. The parameters of the DGL system are those of Fig. 2.

previous section. In Fig. 2 a typical bifurcation curve having codimension 1 is depicted for the on-site mode shown in Table I(a). The numerically obtained bifurcation curve is in very good agreement with the asymptotically obtained one [given by Eq. (23)] in the high-confinement limit $(A \gg 1)$.

A stable background is a necessary requirement for the stability of a bright DGL soliton. We have seen that the zero solution is linearly stable against any type of perturbation when Eq. (6) is satisfied. In addition, stability requires a subcritical bifurcation of the zero solution with the soliton followed by a saddle-node bifurcation. Such a sequence of 
bifurcations is possible when $p_{i}<0$ and $q_{i}>0$. From the approximate expressions for highly confined dissipative solitons, the saddle-node bifurcation of the on-site mode happens for $\epsilon \simeq-p_{i}^{2} / 4 q_{i}$. Notice that the aforementioned conditions are necessary but not sufficient for the stability of the solutions. Thus, one can look for stable solitons in the upper branch of the bifurcation curve, for values of $\epsilon$ that lie approximately in the region $\left(-p_{i}^{2} / 4 q_{i},-4\left|\alpha_{i}\right|\right)$.

In Fig. 3 typical examples of numerically obtained soliton solutions are shown. We have tested the stability by performing a series of numerical simulations for all the modes shown in Table I and for different values of the parameters. The parameter space of the DGL equation chosen is such that the single-peak (on-site) mode is stable. Our simulations revealed that three of the modes are always stable, whereas the remaining three are unstable. More specifically, the singlepeak soliton is selected to be stable, one of the two doublepeaked modes is stable, and one of the three four-peaked modes is also stable.

The dynamics of the unstable modes can lead to different scenarios. For example, for the parameters shown in Fig. 2 and for maximum intensity $A^{2}=1.9$, all of the unstable modes transform to mode (a), i.e., (a) $\rightarrow$ (a), (b) $\rightarrow$ (a), (c) $\rightarrow$ (c), (d) $\rightarrow$ (a), (e) $\rightarrow$ (a), (f) $\rightarrow$ (f). According to the values of the parameters and the perturbations applied, different instability dynamics occurred. However, we noticed that as the parameters $p_{i}$ and $q_{i}$ become larger, there is a trend for all $n$-peaked solutions to transform to the corresponding stable $n$-peaked solution. For example, for $p=1-3 i, q=1+(1 / 3) i$, and for maximum soliton amplitude $A=2.6$, the instability dynamics result in (a) $\rightarrow$ (a), (b) $\rightarrow$ (c), (c) $\rightarrow$ (c), (d) $\rightarrow$ (f), (e) $\rightarrow$ (f), (f) $\rightarrow$ (f) .

\section{CONCLUSIONS}

In conclusion, we have considered a two-dimensional dissipative waveguide lattice which is modeled by a twodimensional DGL equation. We have studied the linear discrete diffraction and gain properties of the system. In the nonlinear case, families of dissipative discrete soliton solutions have been identified, both numerically and asymptotically in the case of high confinement, and their stability has been examined numerically.
[1] M. C. Cross and P. C. Hohenberg, Rev. Mod. Phys. 65, 851 (1993).

[2] I. S. Aranson and L. Kramer, Rev. Mod. Phys. 74, 99 (2002).

[3] N. Akhmediev and A. Ankiewicz, Solitons, Nonlinear Pulses and Beams (Chapman and Hall, London, 1997).

[4] L. Hocking and K. Stewartson, Proc. R. Soc. London, Ser. A 326, 289 (1972).

[5] N. Pereira and L. Stenflo, Phys. Fluids 20, 1733 (1977).

[6] K. Nozaki and N. Bekki, J. Phys. Soc. Jpn. 53, 1581 (1984).

[7] D. N. Christodoulides, F. Lederer, and Y. Silberberg, Nature (London) 424, 817 (2003).

[8] D. N. Christodoulides and R. I. Joseph, Opt. Lett. 13, 794 (1988).

[9] H. S. Eisenberg, Y. Silberberg, R. Morandotti, A. R. Boyd, and J. S. Aitchison, Phys. Rev. Lett. 81, 3383 (1998).

[10] J. W. Fleischer, M. Segev, N. K. Efremidis, and D. N. Christodoulides, Nature (London) 422, 147 (2003).

[11] N. K. Efremidis, S. Sears, D. N. Christodoulides, J. W. Fleischer, and M. Segev, Phys. Rev. E 66, 046602 (2002).

[12] J. W. Fleischer, T. Carmon, M. Segev, N. K. Efremidis, and D. N. Christodoulides, Phys. Rev. Lett. 90, 023902 (2003).

[13] S. Wang and H. Winful, Appl. Phys. Lett. 52, 1774 (1988).

[14] H. Winful and S. Wang, Appl. Phys. Lett. 53, 1894 (1988).

[15] K. Otsuka, Nonlinear Dynamics in Optical Complex Systems (KTK Scientific Publishers, Tokyo, 1999).

[16] K. Otsuka, Phys. Rev. Lett. 65, 329 (1990).

[17] H. G. Winful and L. Rahman, Phys. Rev. Lett. 65, 1575 (1990).

[18] H. Willaime, O. Cardoso, and P. Tabeling, Phys. Rev. Lett. 67, 3247 (1991).

[19] N. K. Efremidis and D. N. Christodoulides, Phys. Rev. E 67,
026606 (2003).

[20] N. K. Efremidis and D. N. Christodoulides, in Dissipative Solitons, edited by N. Akhmediev and A. Ankiewicz, Lecture Notes in Physics Vol. 661 (Springer-Verlag, Berlin, 2005), pp. 309-325.

[21] N. K. Efremidis and D. N. Christodoulides, Opt. Lett. 29, 2485 (2004).

[22] E. J. Bochove, P. K. Cheo, and G. G. King, Opt. Lett. 28, 1200 (2003).

[23] K. Hizanidis, S. Droulias, I. Tsopelas, N. K. Efremidis, and D. N. Christodoulides, Phys. Scr., T 107, 13 (2004).

[24] E. A. Ultanir, G. I. Stegeman, and D. N. Christodoulides, Opt. Lett. 29, 845 (2004).

[25] M. Ablowitz and J. Ladik, J. Math. Phys. 17, 1011 (1976).

[26] K. Maruno, A. Ankiewicz, and N. Akhmediev, Opt. Commun. 221, 199 (2003).

[27] K. Maruno, A. Ankiewicz, and N. Akhmediev, Phys. Lett. A 347, 231 (2005).

[28] F. Abdullaev, A. Abdumalikov, and B. Umarova, Phys. Lett. A 305, 371 (2002).

[29] U. Peschel, O. Egorov, and F. Lederer, Opt. Lett. 29, 1909 (2004)

[30] J. F. Ravoux, S. Le Dizès, and P. Le Gal, Phys. Rev. E 61, 390 (2000).

[31] A. Mohamadou, A. K. Jiotsa, and T. C. Kofané, Phys. Rev. E 72, 036220 (2005).

[32] N. K. Efremidis and D. N. Christodoulides, Opt. Commun. 246, 345 (2005).

[33] J. Eilbeck, P. Lomdahl, and A. Scott, Physica D 16, 318 (1985).

[34] J. B. Page, Phys. Rev. B 41, 7835 (1990). 